\title{
Phonon-Induced Dephasing of Optically Driven Exciton States in Quantum Dots: Spectral Interpretation
}

\author{
P. MACHNIKOWSKI AND L. JACAK \\ Institute of Physics, Wrocław University of Technology \\ Wybrzeże Wyspiańskiego 27, 50-370 Wrocław, Poland
}

\begin{abstract}
We discuss phonon-induced perturbation of optically driven coherent dynamics of a confined exciton in a quantum dot in terms of nonlinear spectra of the driving pulse. Damping of pulse-area-dependent Rabi oscillations and phonon-assisted pumping of optically inactive states are analyzed.
\end{abstract}

PACS numbers: 78.67.Hc, 63.20.Kr, 03.65.Yz

\section{Introduction}

In recent years it has become possible to control the charge state of a single quantum dot (QD) far beyond the linear absorption regime. A convincing example of this experimental progress are pulse area-dependent Rabi oscillations of the exciton occupation, i.e., coherent transitions from the ground crystal state to the single- or bi-exciton state and back, driven by optical pulses of variable intensity [1]. These achievements show that the atomic-like properties of charge systems confined in a QD may allow one to transfer quantum control schemes developed in the quantum-optical context to the semiconductor systems.

However, charges in QDs, unlike those in natural atoms, undergo strong interactions with their solid-state environment resulting in strong decoherence of their quantum states. In self-assembled dots, an important contribution to decoherence is that of pure dephasing due to carrier-phonon interactions [2, 3]. These phonon-related effects may be analyzed for arbitrary driving pulses within the theoretical approach developed recently $[4,5]$, which allows one to treat phonon effects perturbatively, while unperturbed system evolution, including external driving, is included exactly. 
In this paper we propose a transparent spectral interpretation of the interaction between phonons and carriers in the strongly non-linear regime with the usual spectral density of the phonon reservoir and properly defined nonlinear spectra of control pulses. In this way, damping of Rabi oscillations may be interpreted in terms of a resonance between an oscillating charge density and phonon modes [6]. The same approach will also be used to study the system dynamics upon strong driving in the presence of higher excited states. We will describe a new effect of phonon-assisted pumping of optically inactive levels under appropriate driving conditions.

\section{The system}

We consider a single QD in which a single exciton may be created by a resonantly coupled and appropriately polarized laser pulse. Apart from the optically active ground state the exciton has confined excited states which do not couple to the laser field. The corresponding Hamiltonian reads

$$
H_{\mathrm{QD}}=\sum_{n} \epsilon_{n}|n\rangle\langle n|+\frac{1}{2} f(t)[\exp (\mathrm{i} \omega t)|1\rangle\langle 0|+\text { h.c. }],
$$

where $|0\rangle$ is the ground state of the crystal (empty dot), $|1\rangle$ is the exciton ground state, $|n\rangle$ (for $n \geq 2$ ) are optically inactive excited states, $\epsilon_{n}$ are the energies of the exciton states, and $f(t)$ is the (real) envelope of the laser pulse.

The lattice subsystem is described by the usual Hamiltonian $H_{\mathrm{ph}}=$ $\sum_{\boldsymbol{k}, s} \hbar \omega_{\boldsymbol{k}, s} b_{\boldsymbol{k}, s}^{\dagger} b_{\boldsymbol{k}, s}$, where $\omega_{\boldsymbol{k}, s}$ is the frequency of the phonon mode in branch $s$ with wave vector $\boldsymbol{k}$ and $b_{\boldsymbol{k}, s}^{\dagger}, b_{\boldsymbol{k}, s}$ are the corresponding bosonic creation and annihilation operators. In our discussion only two phonon branches will appear: longitudinal acoustic (LA) with linear dispersion around the center of the Brillouin zone $\omega_{\boldsymbol{k} \text {, LA }}=c_{\mathrm{l}} k$, where $c_{\mathrm{l}}$ is the speed of longitudinal sound, and nearly dispersionless longitudinal optical (LO) for which we put $\omega_{\boldsymbol{k}, \mathrm{LO}}=\Omega$.

The two subsystems are coupled via interaction channels, out of which deformation potential coupling to LA phonons and Fröhlich coupling to the polarization field associated with LO phonons are of most importance for the present discussion. The coupling Hamiltonian is

$$
H_{\mathrm{int}}=\sum_{n, n^{\prime}}|n\rangle\left\langle n^{\prime}\right| \sum_{\boldsymbol{k}, s} F_{n n^{\prime}}^{(s)}(\boldsymbol{k})\left(b_{\boldsymbol{k}, s}^{\dagger}+b_{-\boldsymbol{k}, s}\right),
$$

with coupling constants $[7,8] F_{1 l}^{(\mathrm{LA})}(\boldsymbol{k})=\sqrt{\hbar k /\left(2 \varrho V c_{1}\right)}\left(\sigma_{e}-\sigma_{h}\right) \mathcal{F}_{1 l}(\boldsymbol{k})$ and $F_{1 l}^{(\mathrm{LO})}(\boldsymbol{k})=(e / k) \sqrt{\hbar \Omega /\left(2 V \epsilon_{0} \tilde{\epsilon}\right)} \mathcal{F}_{1 l}(\boldsymbol{k})$, for $l \geq 2$. Here $\epsilon_{0}$ is the vacuum dielectric constant, $\tilde{\epsilon}$ is the low-frequency (lattice) part of the relative dielectric constant, $\varrho$ is the crystal density, and $V$ is the normalization volume for the phonon modes. We have assumed here that the lowest excited states of the exciton are formed by exciting the hole (which has a much larger mass), and we defined the form factors 
$\mathcal{F}_{n n^{\prime}}(\boldsymbol{k})=\int_{-\infty}^{\infty} \mathrm{d}^{3} \boldsymbol{r} \psi_{n}^{*}(\boldsymbol{r}) \exp (\mathrm{i} \boldsymbol{k} \cdot \boldsymbol{r}) \psi_{n^{\prime}}(\boldsymbol{r})$, where $\psi(\boldsymbol{r})$ are single-particle wave functions, assumed to be identical for the electron and the hole. In this model, there is no diagonal coupling to the LO phonons due to exact charge cancellation. On the other hand, the effect of non-diagonal phonon coupling terms for LA phonons is negligible due to a large level spacing, compared to LA energies.

\section{The spectral representation}

The state of the QD subsystem is described by its reduced density matrix $\rho_{\mathrm{QD}}(t)=\operatorname{Tr}_{\mathrm{R}} \rho(t)$, where $\rho(t)$ is the density matrix of the total system and the trace is taken over the reservoir (phonon) degrees of freedom. The density matrix for the total system is found from the standard perturbative expansion with respect to $H_{\text {int }}$ [9]. As discussed in detail in Refs. [8, 10], one then arrives at closed formulae for the diagonal elements of the reduced density matrix, i.e., the occupations of exciton states

$$
\begin{aligned}
\rho_{11} & =\sin ^{2} \frac{\alpha}{2}+\int \mathrm{d} \omega \frac{R_{1}(\omega)}{\omega^{2}} S_{a}(\omega) \\
& -\sum_{l>1} \int \mathrm{d} \omega \frac{R_{l}\left(\omega-\epsilon_{l}\right)}{\omega^{2}}\left[\sin ^{2} \frac{\alpha}{2}+S_{c}(\omega)\right], \\
\rho_{n n} & =\sin ^{2} \frac{\alpha}{2} \int \mathrm{d} \omega \frac{R_{n}\left(\omega-\epsilon_{n}\right)}{\omega^{2}}+\int \mathrm{d} \omega \frac{R_{n}\left(\omega-\epsilon_{n}\right)}{\omega^{2}}\left[S_{b}(\omega)+S_{c}(\omega)\right] .
\end{aligned}
$$

Here $R_{n}(\omega)$ are effective spectral densities of the phonon reservoir associated with various phonon assisted transitions,

$$
\begin{aligned}
R_{n}(\omega) & =\frac{1}{\hbar^{2}}\left|n_{\mathrm{B}}(\omega)+1\right| \\
\times & \frac{1}{N} \sum_{\boldsymbol{k}, s} F_{1 n}^{(s)}(\boldsymbol{k}) F_{n 1}^{(s) *}(\boldsymbol{k})\left[\delta\left(\omega-\omega_{s, \boldsymbol{k}}\right)+\delta\left(\omega+\omega_{s, \boldsymbol{k}}\right)\right],
\end{aligned}
$$

where $n_{\mathrm{B}}(w)$ is the Bose distribution function. These functions depend on the material parameters. The function $R_{1}(\omega)$ corresponds to the LA phonon sideband of the fundamental optical transition in a QD observed in optical experiments.

On the other hand, the nonlinear spectral properties of the optically induced dynamics are contained in the family of spectral functions

$$
\begin{aligned}
& S_{a}(\omega)=\frac{1}{4}\left\{(\cos \alpha)\left|K_{\mathrm{s}}^{(1)}(\omega)\right|^{2}-(\sin \alpha) \operatorname{Re}\left[K_{\mathrm{s}}^{(1) *}(\omega) K_{\mathrm{c}}^{(1)}(\omega)\right]\right\}, \\
& S_{b}(\omega)=\left(\cos ^{2} \frac{\alpha}{2}\right)\left|K_{\mathrm{s}}^{(1 / 2)}(\omega)\right|^{2}-\frac{1}{2}(\sin \alpha) \operatorname{Re}\left[K_{\mathrm{s}}^{(1 / 2) *}(\omega) K_{\mathrm{c}}^{(1 / 2)}(\omega)\right], \\
& S_{c}(\omega)=\left(\sin ^{2} \frac{\alpha}{2}\right)\left|K_{\mathrm{s}}^{(1 / 2)}(\omega)\right|^{2}+\frac{1}{2}(\sin \alpha) \operatorname{Re}\left[K_{\mathrm{s}}^{(1 / 2) *}(\omega) K_{\mathrm{c}}^{(1 / 2)}(\omega)\right],
\end{aligned}
$$

with 


$$
\begin{aligned}
& K_{\mathrm{s}}^{(\mu)}(\omega)=\int_{-\infty}^{\infty} \mathrm{d} \tau \exp (\mathrm{i} \omega \tau) \frac{\mathrm{d}}{\mathrm{d} \tau} \sin (\mu \Phi(\tau)), \\
& K_{\mathrm{c}}^{(\mu)}(\omega)=\int_{-\infty}^{\infty} \mathrm{d} \tau \exp (\mathrm{i} \omega \tau) \frac{d}{d \tau} \cos (\mu \Phi(\tau)),
\end{aligned}
$$

where $\Phi(t)=\int_{-\infty}^{t} \mathrm{~d} \tau f(\tau)$, and $\alpha=\Phi(\infty)$ is the pulse area.

\section{Phonon-assisted damping}

Let us start with relatively long driving pulses where only LA phonons contribute to dephasing. We will use InAs/GaAs system parameters: $c_{1}=5150 \mathrm{~m} / \mathrm{s}$, $\varrho=5350 \mathrm{~kg} / \mathrm{m}^{3}, \sigma_{\mathrm{e}}-\sigma_{\mathrm{h}}=9.5 \mathrm{eV}$. The wave functions will be modelled by anisotropic Gaussians with extension $l_{\perp}=5 \mathrm{~nm}$ in-plane and $l_{z}=2 \mathrm{~nm}$ along the growth axis. The pulse is assumed to have a Gaussian envelope $f(t)=\exp \left(\left(t / \tau_{0}\right)^{2} / 2\right) /\left(\sqrt{2 \pi} \tau_{0}\right)$. The final occupation of the confined exciton state as a function of the pulse area is shown in Fig. 1. The striking feature is that damping decreases both for short and long pulses. This can be understood with the help of Eq. (3) if one realizes that the spectral function $S_{a}$ defined by Eqs. (6) and (9) scales according to $S_{a}(\omega)=\tilde{S}_{a}\left(\omega \tau_{0}\right)$, where $\tilde{S}_{a}(x)$ is a certain function

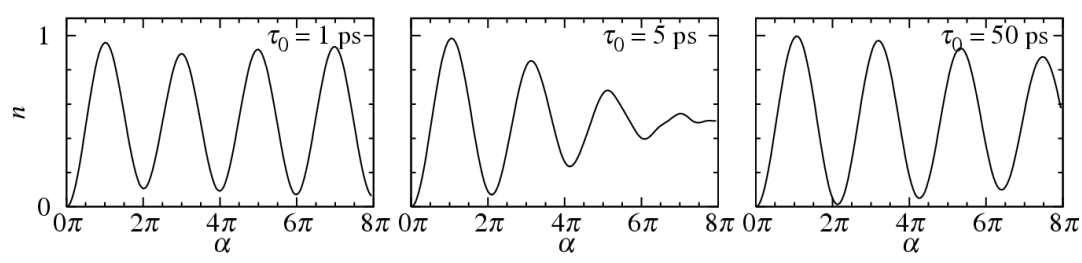

Fig. 1. Pulse area dependent Rabi oscillations for three pulse durations.
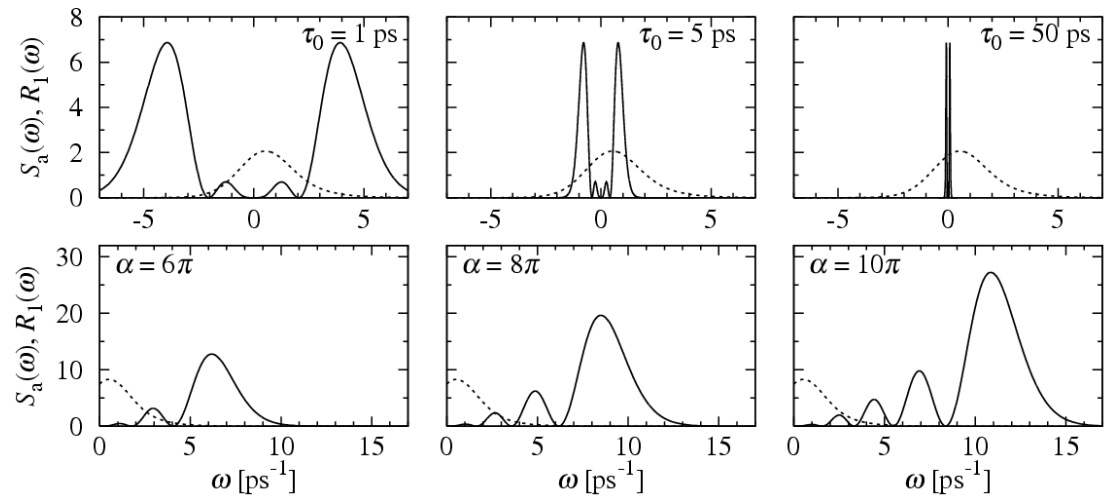

Fig. 2. Nonlinear spectra of the optical pulse (solid) and the phonon spectral density (dotted). Upper: for $\alpha=4 \pi$ and pulse durations as in Fig. 1. Lower: for $\tau_{0}=1$ ps and various pulse areas $\alpha$. 
dependent only on the pulse area $\alpha$ (for a given general pulse shape). As can be seen in Fig. 2 (upper), both for short and long pulses the overlap with the phonon spectral density $R_{1}(\omega)$ is reduced.

Nonlinear spectra for larger pulse areas $\alpha$ (Fig. 2, lower) show a series of maxima of growing magnitude that develop without a considerable modification of the low frequency sector of the spectrum. The strongest interaction with phonons appears when the largest maximum overlaps with the phonon spectral density, i.e., when $\alpha / \tau_{0} \simeq \omega_{0}$, where $\omega_{0} \simeq c_{1} / l_{\perp}$ is the characteristic frequency of the effectively coupled LA phonon modes. This is easily understood on the semiclassical level by invoking the picture of periodically appearing and disappearing charge distribution in the dot which acts (via carrier-phonon couplings) as an oscillating driving force on the lattice ions. Damping of Rabi oscillations is strong when the oscillations of the driving force are resonant to many effectively coupled phonon modes.

\section{Phonon-assisted pumping of dark levels}

For short (sub-picosecond) pulses a new dynamical effect appears, related to LO phonon excitations. Since the separation between the exciton energy levels is comparable to the LO energy, the carriers cannot adiabatically follow the oscillating force associated with the LO phonons excited by the carrier dynamics and transitions may occur, leading to non-vanishing population of optically inactive exciton states.

Here we will consider a single excited state with the energy of $E=16 \mathrm{meV}$, formed by exciting the hole (this is justified by numerical diagonalization [7]). For calculations we take $\tilde{\epsilon}=70$ and $\Omega=54 \mathrm{ps}^{-1}$. The occupation of this state is given by Eq. (4). The first term in this expression reflects the mixing of exciton states to polaronic effects [11], while the second one describes the dynamical effect of interest. The spectral density of LO phonons is peaked at $\omega=\Omega$, so that the effect is governed by the value of the nonlinear spectral function $S_{b}(\omega)+S_{c}(\omega)$ at $\omega=E / \hbar+\Omega$, which reflects the fact that the optically inactive state may only be occupied via a phonon-assisted process.
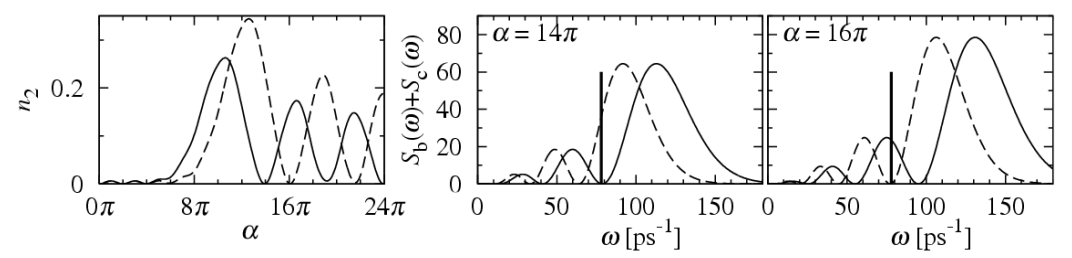

Fig. 3. Left: phonon-assisted occupation of an optically inactive exciton level. Right: the relevant nonlinear spectra (the bar shows the frequency corresponding to the phonon-assisted transition). Solid line corresponds to $\tau_{0}=0.065 \mathrm{ps}$ and dashed one to $\tau_{0}=$ $0.08 \mathrm{ps}$. 
The occupation of the excited state is shown for two pulse durations in Fig. 3. The occupation is high when the (almost discrete) transition energy coincides with a maximum of the nonlinear spectrum. Therefore, the scaling property of nonlinear spectra leads to an oscillating dependence of the occupation on the pulse duration. Moreover, a shift of nonlinear spectra with growing pulse area (see Fig. 3, right) leads to oscillating dependence on the latter. Remarkably, for sufficiently strong pulses the occupation of the excited state is considerable already for pulses of duration $\sim 100$ fs.

\section{Conclusion}

We have discussed phonon-induced damping of Rabi oscillations in quantum dots. We have shown that the phonon perturbation results from a resonance between the optically driven dynamics of confined carriers and phonon modes. The damping effect is strong when the Rabi frequency, with which the carriers appear and disappear in the $\mathrm{QD}$, corresponds to the most effectively coupled phonons. We have shown also that nonadiabaticity of the carrier dynamics with respect to the LO lattice oscillations leads to phonon-assisted occupation of excited states that are otherwise optically inactive.

The presented results shed light on the mechanisms of interaction between the carriers undergoing coherently driven dynamics in a semiconductor QD and their phonon environment. In this way, they contribute to the understanding of the current experimental results by extending the knowledge available from numerical solutions [12]. The spectral interpretation provides also a "thumb rule" for predicting the magnitude of the dephasing effect under various driving conditions. The spectral picture shows also that phonon-assisted excitations may be avoided in the limit of slow (adiabatic) driving [5]. They may also be reduced, to some extent, if favorable nonlinear spectra are engineered by pulse-shaping [13].

\section{Acknowledgment}

Supported by the Polish Ministry of Scientific Research and Information Technology (PBZ-MIN-008/P03/2003 and PB 2 P03B 08525). P.M. is grateful to A. von Humboldt Foundation for support.

\section{References}

[1] A. Zrenner, E. Beham, S. Stufler, F. Findeis, M. Bichler, G. Abstreiter, Nature 418, 612 (2002).

[2] B. Krummheuer, V.M. Axt, T. Kuhn, Phys. Rev. B 65, 195313 (2002).

[3] A. Vagov, V.M. Axt, T. Kuhn, Phys. Rev. B 67, 115338 (2003).

[4] R. Alicki, M. Horodecki, P. Horodecki, R. Horodecki, Phys. Rev. A 65, 062101 (2002), quant-ph/0105115. 
[5] R. Alicki, M. Horodecki, P. Horodecki, R. Horodecki, L. Jacak, P. Machnikowski, Phys. Rev. A 70, 010501(R) (2004), quant-ph/0302058.

[6] P. Machnikowski, L. Jacak, Phys. Rev. B 69, 193302 (2004), cond-mat/0305165.

[7] L. Jacak, P. Machnikowski, J. Krasnyj, P. Zoller, Eur. Phys. J. D 22, 319 (2003).

[8] A. Grodecka, L. Jacak, P. Machnikowski, K. Roszak, in: Quantum Dots: Research Developments, Ed. P.A. Ling, Nova Science, Hauppauge (NY), in press, condmat/0404364.

[9] C. Cohen-Tannoudji, J. Dupont-Roc, G. Grynberg, Atom-Phonon Interactions, Wiley-Interscience, New York 1998.

[10] K. Roszak, A. Grodecka, P. Machnikowski, T. Kuhn, Phys. Rev. B 71, 195333 (2005)

[11] L. Jacak, J. Krasnyj, D. Jacak, P. Machnikowski, Phys. Rev. B 65, 113305 (2002); 67, 035303 (2003).

[12] J. Förstner, C. Weber, J. Danckwerts, A. Knorr, Phys. Rev. Lett. 91, 127401 (2003).

[13] V.M. Axt, P. Machnikowski, T. Kuhn, Phys. Rev. B 71, 155305 (2005), cond-mat/0408322. 